\title{
Multi-layered CdSe/ZnS/CdSe heteronanocrystals to generate and tune white light
}

\author{
Sedat Nizamoglu, Evren Mutlugun, Tuncay Ozel, and Hilmi Volkan Demir \\ Department of Electrical and Electronics Engineering, Department of Physics, Nanotechnology Research Center and Institute of Materials \\ Science and Nanotechnology, Bilkent University, Ankara, 06800 Turkey
}

Sameer Sapra, Nikolai Gaponik, and Alexander Eychmüller

Physikalische Chemie, TU Dresden, Bergstr. 66b, 01062 Dresden, Germany

Till date different types of white light emitting diodes (WLEDs) including multi-chip WLEDs, monolithic WLEDs, and color-conversion WLEDs have been investigated [1-3]. Among them, color-conversion WLEDs have been the most widely investigated thus far and are commercially used today [4]. For WLEDs applications, recently semiconductor quantum dot nanocrystals $(\mathrm{NCs})$ have also attracted great attention because of their tunable photoluminescence, high photoluminescence quantum yields, high photostability, and easy handling. Nanocrystals have been exploited in several WLED implementations to date [5-10]. However, in all of these NC based WLEDs mono-color emitting nanocrystals or their multiple combinations have been used as the luminophors. Only recently multi-color emitting semiconductor heteronanocrystals (hetero-NCs) have been introduced by synthesizing a quantum-dot-quantum-well (QDQW) structure in CdSe-ZnS material system [11]. In this heterostructure, first a CdSe core (quantum well), then a surrounding $\mathrm{ZnS}$ shell (quantum barrier), and finally a CdSe shell (quantum well) are synthesized one after the other. By using these multi-layered CdSe-ZnS hetero-NCs in solution, white light generation with multi-color emission from their CdSe cores (in yelloworange) and from their CdSe shells (in cyan) has been shown [12]. However, for white light generation, using these heteronanocrystals in solution is not sufficient for the potential application in solid state lighting. Therefore, optical properties of such heteronanocrystal luminophors in the solid form need to be carefully analyzed to understand and control the photometric properties of the generated white light.

In this study, we present tuneable white light generation by controlling $\mathrm{CdSe} / \mathrm{ZnS} / \mathrm{CdSe}$ core/shell/shell heteronanocrystals integrated on InGaN/GaN light emitting diodes [13-15]. These multi-layered quantum dots, also known as onion-like heterostructures, are designed and synthesized to emit in red (around $600 \mathrm{~nm}$ ) from the $\mathrm{CdSe}$ core and in green (around $550 \mathrm{~nm}$ ) from the CdSe shell. By designing and hybridizing these red-green emitting heterostructures on blue emitting LEDs, we demonstrate integrated WLEDs on a single chip. By controlling the number of integrated heteronanocrystals, their $(\mathrm{x}, \mathrm{y})$ tristimulus coordinates are tuned from $(0.26,0.23)$ to $(0.37,0.36)$ as shown in figure $1(\mathrm{a})$, along with their corresponding correlated color temperature tuned from $27413 \mathrm{~K}$ to $4192 \mathrm{~K}$ and the luminous efficacy of their optical radiation (the ratio of the emitted luminous flux to the radiant flux) tuned from $258 \mathrm{~lm} / \mathrm{W}$ to $375 \mathrm{~lm} / \mathrm{W}$. We further investigate the change of infilm optical properties of these heteronanocrystals with respect to their in-solution emission. This plays a significant role in the use of these structures in hybrid LED applications.

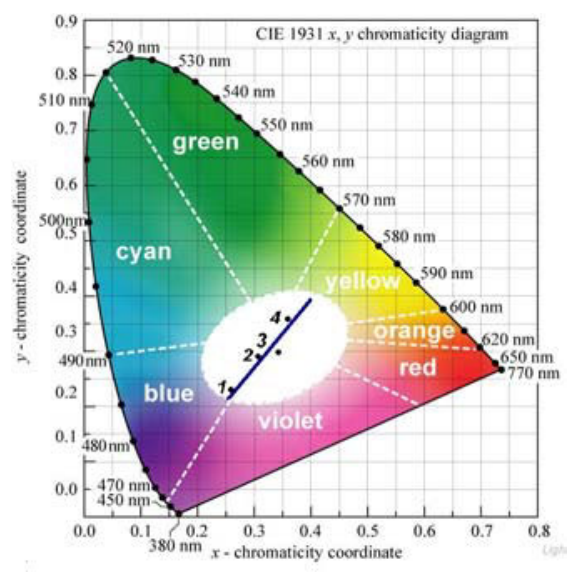

(a)

\begin{tabular}{|l|l|l|l|l|l|}
\hline $\begin{array}{l}\text { Hetero- } \\
\text { NC-WLED }\end{array}$ & $\begin{array}{l}\text { Number of } \\
\text { nanocrystals } \\
\text { (nmol) }\end{array}$ & $\mathrm{X}$ & $\mathrm{Y}$ & $\begin{array}{l}\text { Correlated } \\
\text { color } \\
\text { temperature } \\
\text { (K) }\end{array}$ & $\begin{array}{l}\text { Optical } \\
\text { luminous } \\
\text { efficacy } \\
\text { (lm/W) }\end{array}$ \\
\hline 1 & 0.33 & 0.26 & 0.23 & 27413 & 258 \\
\hline 2 & 0.40 & 0.31 & 0.29 & 6780 & 313 \\
\hline 3 & 0.46 & 0.34 & 0.30 & 4635 & 315 \\
\hline 4 & 0.50 & 0.37 & 0.36 & 4192 & 375 \\
\hline
\end{tabular}

(b)

Figure 1. (a) Tuning shades of the generated white light across $\mathrm{CIE}$ (1931) chromaticity diagram, and (b) photometric characteristics of our hybrid white hetero-NC-WLEDs (1-4).

For sample hetero-NC-WLED 1, we integrate $0.33 \mathrm{nmol}$ heteronanocrystals on the blue LED and obtain the tristimulus coordinates of $(0.26,0.23)$ with a color temperature of $27413 \mathrm{~K}$, and a luminous efficacy of the 
emitted spectrum of $258 \mathrm{~lm} / \mathrm{W}$. This operating point falls in the white region as shown in Figure 1 (a). From hetero-NC based WLED 1 to 4, we gradually increase the integrated number heteronanocrystals on the pumping LED platform. Since the relative emission of the heteronanocrystals increases with respect to the blue LED electroluminescence, the color temperature decreases. In hetero-NC-WLED 4, to obtain a warmer white light, we hybridize $0.50 \mathrm{nmol}$ heteronanocrystals, which yields an operating point with the tristimulus coordinates of $(0.50,0.37)$ in the white region, a correlated color temperature of $4192 \mathrm{~K}$, and a high luminous efficacy of the emitted spectrum of $375 \mathrm{~lm} / \mathrm{W}$. Photometric characteristics of our hybrid white hetero-NC-WLEDs are further summarized in Figure 1 (b).

A variety of physical mechanisms including reabsorption, dipole-dipole interaction, energy transfer, and effective dielectric constant change of the environment affect the optical emission properties of these heteronanocrystals. To investigate these luminescence modifications of such heteronanocrystals, we use another set of heteronanocrystals with in-solution luminescence peaks around $613 \mathrm{~nm}$ coming from CdSe core and around $558 \mathrm{~nm}$ coming from the shell. For investigating in-film luminescence red shift with respect to insolution luminescence, we prepare four samples of heteronanocrystal films with different heteronanocrystal amounts precisely in integer multiples (1, 2, 4, and 9 multiples) of the starting sample (ca. $1 \mathrm{nmol}$ ). Tables 1(a) and 1(b) exhibit the photoluminescence (PL) peak wavelengths and relative peak intensities of these samples (Samples 1-4). These samples are all excited with a He-Cd laser at $325 \mathrm{~nm}$ at room temperature. By increasing the number of heteronanocrystals, we observe that both emission peaks shift towards red in the film and that the relative intensities of emission at shorter wavelengths decrease. In this red shift, re-absorption, dipole-dipole interaction, and energy transfer are the three mainly effective processes. However, with respect to the in-solution PL (reference), the core emission in the thin films (Samples 1-3) makes a blue shift as shown in Table 1(a), which is attributed to the effect of the substrate. This effect decreases with increasing number of nanocrystals [16]. Furthermore, for Samples 1-4, it is interesting to note that, although the peak emission from the core makes a red shift of $8.2 \mathrm{~nm}$ for Sample 1 to 4 , the peak emission from the shell reveals a red shift of $11.0 \mathrm{~nm}$. Thus, the shells exhibit a larger red shift in comparison to the core in the thin films. The additional red shift of shell in comparison with the core is due to delocalization of excitons in the shell over other coupled shells. At this point, it is also worth discussing that, though these onion-like heteronanocrystals have been previously [11] and also in our works [13-15] shown to exhibit multi-color emission in solutions and in films, a further study on a singlenanocrystal level needs to be conducted to verify the multiple nature of this emission from single nanocrystals. However, these present discussions will still remain valid regardless the single heteronanocrytals exhibit multiple color emission or not.

Table 1. (a) Photoluminescence peak wavelengths and (b) photoluminescence relative peak intensities of the synthesized onion-like $(\mathrm{CdSe}) \mathrm{ZnS} / \mathrm{CdSe}$ heteronanocrystals in solution and in film (Samples 1-4).

\begin{tabular}{|l||l||c|}
\hline \multicolumn{1}{|c||}{ Peak wavelengths (nm) } & $\begin{array}{c}\text { Shell } \\
\text { emission } \\
\text { peak }\end{array}$ & $\begin{array}{c}\text { Core } \\
\text { emission } \\
\text { peak }\end{array}$ \\
\hline Reference (in solution) & 558.42 & 613.32 \\
\hline Sample 1 (in film) & 561.75 & 602.17 \\
\hline Sample 2 (2x hetero-NCs in Sample 1) & 562.74 & 604.64 \\
\hline Sample 3 (4 x hetero-NCs in Sample 1) & 560.48 & 612.62 \\
\hline Sample 4 (9x hetero-NCs in Sample 1) & 569.44 & 621.53 \\
\hline
\end{tabular}

\begin{tabular}{|l|l|l|}
\hline Ramples & $\begin{array}{c}\text { Rhell } \\
\text { emission } \\
\text { peak }\end{array}$ & $\begin{array}{c}\text { Core } \\
\text { emission } \\
\text { peak }\end{array}$ \\
\hline Reference (in solution) & 0.695 & 0.156 \\
\hline Sample 1 (in film) & 0.573 & 0.152 \\
\hline Sample 2 (2x hetero-NCs in Sample 1) & 0.609 & 0.145 \\
\hline Sample 3 (4x hetero-NCs in Sample 1) & 0.228 & 0.668 \\
\hline Sample 4 (9x hetero-NCs in Sample 1) & 0.136 & 0.820 \\
\hline
\end{tabular}

In conclusion, we fabricated hybrid white light emitting diodes by integration of $\mathrm{CdSe} / \mathrm{ZnS} / \mathrm{CdSe}$ core/shell/shell heteronanocrystals on blue emitting InGaN/GaN LEDs. We tuned white light parameters of (x,y) tristimulus coordinates from $(0.26,0.23)$ to $(0.37,0.36)$, correlated color temperature from 27413 to $4192 \mathrm{~K}$, and luminous efficacy of optical radiation from 258 to $375 \mathrm{~lm} / \mathrm{W}$ by controlling the number of integrated heteronanocrystals. Also, we further investigated modifications of in-film optical properties of these heteronanocrystals with respect to their in-solution emission, which plays an important role in designing solid heteronanocrytal luminophors in solid state lighting.

Acknowledgements: This work is supported by EU NoE PHOREMOST 511616 and Marie Curie IRG MOON 021391 and TUBITAK under the Project No. EEEAG 106E020, 104E114, 107E088, 107E297, 105E065, and 105E066. HVD also acknowledges additional support from TUBA GEBIP and ESF EURYI programs.

References

[1] E. F. Schubert, Cambridge University Press, 2006.

[2] M. Yamada et al., IEICE Trans. Electron. E88-C, 9, 1860, 2005.

[3] H. Chen et al, IEEE Photon. Technol. Lett., 18, 1430, 2006.

[4] S. Nakamura and G. Fasol, Berlin: Springer, 1997.

[5] S. Nizamoglu et al., Nanotechnology, 18, 065709, 2007.

[6] S. Nizamoglu et al., Appl. Phys. Lett., 92, 031102, 2008.

[7] H. V. Demir et al., New J. Phys., 9, 362, 2007.

[8] S. Nizamoglu et al., J. Opt. A: Pure Appl. Opt., 9, S419, 2007.
[9] S. Nizamoglu et al., Nanotechnology, 18, 405702, 2007.

[10] M. Ali et al., Nanotechnology, 18, 075401, 2007.

[11] D. Battaglia et al., J. Am. Chem. Soc., 127, 10889, 2005.

[12] S. Sapra et al., Adv. Mater., 19, 569, 2007.

[13] S. Nizamoglu et al., Appl. Phys. Lett., 92, 113110, 2008.

[14] S. Nizamoglu et al., Opt. Exp., 16, 3515, 2008.

[15] H. V. Demir et al., Nanotechnology, 19, 3352032008.

[16] A. Chistyakov et al., Laser Phys., 16, 1625, 2006. 